\title{
ON CONVEXIFICATION OF SOME MINIMUM DISTANCE PROBLEMS
}

\author{
G. Chesi*, A. Tesi $\#$, A. Vicino* and R. Genesio\# \\ * Dipartimento di Ingegneria dell'Informazione, Università di Siena \\ Via Roma 56, 53100 Siena, Italy \\ Fax: ++390577 263602; E-mail: vicino@unisi.it \\ \# Dipartimento di Sistemi e Informatica, Università di Firenze \\ Via di S. Marta 3, 50139 Firenze, Italy \\ Fax: ++39055 4796363; E-mail: atesi@dsi.unifi.it
}

Keywords : Optimization, Linear Matrix Inequalities (LMIs), Homogeneous form, Stability, Robustness.

\begin{abstract}
This paper considers the problem of determining the minimum euclidean distance of a point from a polynomial surface in $R^{n}$. It is well known that this problem is in general non-convex. The main purpose of the paper is to investigate to what extent Linear Matrix Inequality (LMI) techniques can be exploited for solving this problem. The first result of the paper shows that a lower bound to the global minimum can be achieved via the solution of a oneparameter family of Linear Matrix Inequalities (LMIs). It is also pointed out that for some classes of problems the solution of a single LMI problem provides the lower bound. The second result concerns the tightness of the bound. It is shown that optimality of the lower bound can be easily checked via the solution of a system of linear equations. Two application examples are finally presented to show potentialities of the approach.
\end{abstract}

\section{Introduction}

It is a well known fact that a large number of system analysis and control problems amount to computing the minimum distance from a point to a surface in a finite dimensional space. Several issues in robustness analysis of control systems, such as the $\mu$ and the related stability margin computation [1]-[3], and the problem of estimating the domain of attraction of equilibria of nonlinear systems [4],[5] fall in this class of problems. Unfortunately, it is also known that the great majority of such problems can be formulated as non-convex optimization programs, whose solution is in general extremely difficult, if not impossible to obtain with reasonable computational effort.

Recently, powerful convex optimization techniques have been devised for problems in the form of Linear Matrix In- equalities (LMIs) [6]. Such techniques have been succesfully employed in connection with suitable changes of variables for convexifying some classes of optimization problems.

The main purpose of this paper is to show how LMI techniques can be exploited to solve some classes of minimum distance problems. More specifically, the problem of determining the minimum euclidean distance of a point from a polynomial surface in $R^{n}$ is considered. It is first shown that a lower bound to the global minimum can be achieved via the solution of a one-parameter family of LMIs, once a suitable change of variables has been performed. In particular, each LMI problem requires the minimization of the maximum eigenvalue of a symmetric matrix. Successively, tightness of this lower bound is investigated, providing a simple optimality test based on the solution of a system of linear equations.

The paper is organized as follows. In Section 2 the minimum distance problem is formulated and its canonical form is introduced. The lower bound based on LMI techniques is given in Section 3. Section 4 contains the optimality test for the lower bound. Two application examples are given in Section 5 to illustrate the features of the approach. Some concluding comments end the paper in Section 6.

\section{Notation.}

$R^{n}$ : real $n$-space;

$R_{0}^{n}: R^{n} \backslash \emptyset$

$x=\left(x_{1}, \ldots, x_{n}\right)^{\prime}$ : vector of $R^{n} ;$

$R^{n \times n}:$ real $n \times n$-space;

$A=\left[a_{i j}\right]:$ matrix of $R^{n \times n}$;

$A^{\prime}, A^{-1}$ : transpose of $A$, inverse of $A$;

$\operatorname{det}[A]$ : determinant of matrix $A$;

$\operatorname{Ker}[A]$ : null space of matrix $A$;

$E_{n}: n \times n$ identity matrix;

$A>0(A \geq 0)$ : positive definite (semidefinite) matrix;

$\lambda_{M}\{A\}$ : maximum real eigenvalue of matrix $A$;

$\|\cdot\|_{2, W}$ : weighted euclidean norm; 
$H_{n}(A)$ : Hurwitz determinant of order $n$ of matrix $A$ problem characteristic polynomial.

\section{Problem formulation and preliminaries}

In order to state the problem, we need to introduce the following definitions.

\section{Definition 1.}

A map $f^{(d)}: R^{n} \longrightarrow R$ is said a (real $n$-variate homogeneous) form of degree $d$ if

$$
f^{(d)}(x)=f^{(d)}\left(x_{1}, \ldots, x_{n}\right)=\sum_{i_{1} \geq 0, \ldots, i_{n} \geq 0}^{i_{1}+\ldots+i_{n}=d} f_{i_{1}, \ldots, i_{n}} x_{1}^{i_{1}} \cdots x_{n}^{i_{n}}
$$

where $f_{i_{1}, \ldots, i_{n}} \in R$ are the coefficients and $d$ is a nonnegative integer.

With some abuse of notation, we will sometimes denote a form simply by $f$, dropping the explicit dependence on the degree.

\section{Definition 2.}

A (real $n$-variate homogeneous) form $f$ is said positive definite (semidefinite) in a certain region if $f(x)>0$ $(f(x) \geq 0)$ for all $x$ in that region.

A (real $n$-variate homogeneous) form $f$ is said positive definite (semidefinite) if $f(x)>0(f(x) \geq 0)$ for all $x \in R_{0}^{n}$.

Observe that a positive definite form is necessarily of even degree. Moreover, a (real $n$-variate) polynomial $p(x)$ can always be written as the sum of forms of suitable degree, i.e.,

$$
p(x)=p\left(x_{1}, \ldots, x_{n}\right)=\sum_{k=0}^{N} f^{(k)}\left(x_{1}, \ldots, x_{n}\right)
$$

where $f^{(k)}$ are (real $n$-variate homogeneous) forms of degree $k$.

Now, we introduce a definition on polynomials which will be used to avoid the trivial case when the solution of the considered optimization problem is zero.

\section{Definition 3.}

A polynomial $p(x)=\sum_{k=0}^{N} f^{(k)}(x)$ is said locally positive definite if its form of minimum degree is positive definite.

We can now formulate our problem.

\section{Problem I.}

Let $p(x)$ be a locally positive definite polynomial and $W$ a symmetric positive definite matrix. Compute the minimum distance from the origin to the surface defined by $p(x)=0$, i.e., solve the constrained optimization

$$
\rho^{*}=\min _{x \in R_{0}^{n}}\|x\|_{2, W}^{2}
$$

subject to $\quad p(x)=0$.

\section{Remark 1.}

The above optimization problem can incorporate multiple polynomial constraints in an obvious way. Suppose that the constraints are $p_{1}(x)=0$ and $p_{2}(x)=0$. Thus, an equivalent scalar polynomial constraint is $p_{1}^{2}(x)+p_{2}^{2}(x)=0$.

Our aim is to investigate how convex optimization techniques such as LMIs can be exploited for approximating the true solution. To this purpose, we introduce a canonical optimization problem where the constraint is given by a single polynomial consisting of even forms only.

\section{Problem II.}

Let $u(x)$ be a positive definite quadratic form and $w(x)$ be a locally positive definite polynomial of the form

$$
w\left(x_{1}, \ldots, x_{n}\right)=\sum_{k=0}^{m} v^{(2 k)}\left(x_{1}, \ldots, x_{n}\right),
$$

where $v^{(2 k)}$ are given forms of even degree. Solve the following constrained optimization problem

$$
\begin{gathered}
c^{*}=\min _{x \in R_{0}^{n}} u(x) \\
\text { subject to } \quad w(x)=0 .
\end{gathered}
$$

At a first sight, Problem II appears a simplified version of Problem I. However, it is straightforward to show that any problem of the form (3) can be equivalently written in the form of (5).

\section{Proposition 1.}

Let $p(x)$ and $W$ be given. Then, $u(x)$ and $w(x)$ can be constructed such that $\rho^{*}=c^{*}$.

\section{An LMI-based lower bound}

In this section, our primary goal is to provide a lower bound to the optimal solution $c^{*}$ that can be computed via LMI techniques.

Figure 1 shows a geometrical view of the tangency problem (5). Since $w(x)$ is locally positive definite, it turns out that $c^{*}$ can be computed as the smallest positive value of $c$ such that $w(x)$ loses positivity for some $x$ belonging to the level set

$$
\mathcal{U}_{c}=\left\{x \in R^{n}: \quad u(x)=c\right\} .
$$

This fact suggests that the computation of $c^{*}$ could be performed by a sequence of positivity tests of $w(x)$ on the ellipsoids $u(x)=c$. To this purpose, let us consider the 


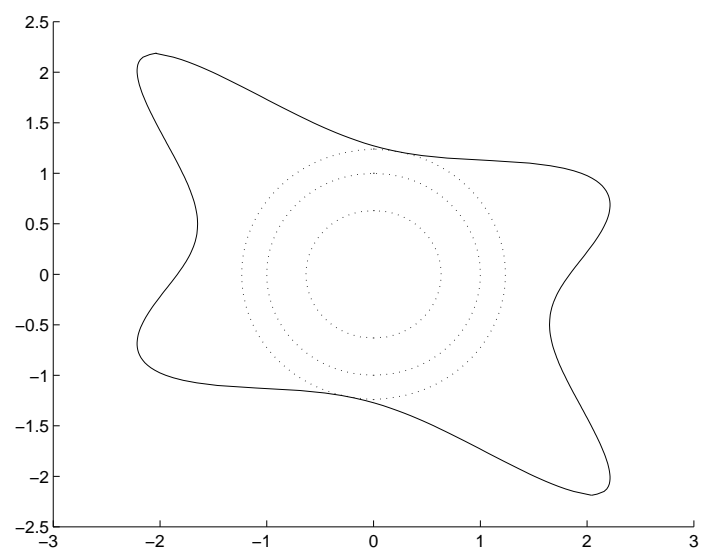

Figure 1: Geometrical view of Problem II.

real form of degree $2 m$

$$
\hat{w}(x ; c)=\sum_{k=0}^{m} v^{(2 k)}(x) \frac{u^{m-k}(x)}{c^{m-k}}
$$

where $c$ is any positive real. We have the following result.

\section{Lemma 1.}

For a given positive $c$, the following two statements are equivalent:
i) $w(x)>0 \quad \forall x \in \mathcal{U}_{c}$;
ii) $\hat{w}(x ; c)>0 \quad \forall x \in R_{0}^{n}$.

Proof.

ii) $\Longrightarrow$ i). Obvious.

i) $\Longrightarrow$ ii). For a given $x \in R_{0}^{n}$, there exists a strictly positive $r$ such that

$$
\tilde{x}=r x \quad \text { and } \quad u(\tilde{x}) \in \mathcal{U}_{c} .
$$

Condition i) implies that $w(\tilde{x})>0$ and by (4), (7) this yields

$$
\hat{w}(\tilde{x} ; c)>0 .
$$

Since $\hat{w}(x ; c)$ is a (homogeneous) form in $x$, one has

$$
\hat{w}(x ; c)=r^{2 m} \hat{w}(\tilde{x} ; c) .
$$

The proof follows from (8), (9) and arbitrariness of $x$.

The lemma above suggests that a constrained polynomial positivity test is equivalent to a positivity test of a form.

Now, we rewrite $\hat{w}(x ; c)$ in a form more convenient for our purposes. To proceed, let $x \in R^{n}$ and consider the sub-vector $x_{[k]} \in R^{n-k+1}$ composed of the last $n-k+1$ coordinates of $x$, i.e.,

$$
x_{[k]}=\left(x_{k}, x_{k+1}, \cdots, x_{n}\right)^{\prime} .
$$

Obviously, $x_{[1]}=x$. Furthermore, let us introduce the vector defined recursively as follows

$$
x_{[k]}^{\{m\}}:= \begin{cases}\left(x_{k} x_{[k]}^{\{m-1\}^{\prime}}, \cdots, x_{n} x_{[n]}^{\{m-1\}^{\prime}}\right)^{\prime} & m>0 \\ 1 & m=0 .\end{cases}
$$

For ease of notation, we denote the vector $x_{[1]}^{\{m\}}$, composed of all the distinct monomials of degree $m$ built with the $n$ coordinates of $x$, by $x^{\{m\}}$. Notice that $x^{\{m\}} \in R^{\sigma(n, m)}$ where

$$
\sigma(n, m):=\left(\begin{array}{c}
n+m-1 \\
n-1
\end{array}\right) .
$$

Since $\hat{w}(x ; c)$ is a real form of degree $2 m$, it is easy to show that

$$
\hat{w}(x ; c)=x^{\{m\}^{\prime}} \Omega_{2 m}(c) x^{\{m\}}
$$

where $\Omega_{2 m}(c) \in R^{\sigma(n, m) \times \sigma(n, m)}$ is a suitable symmetric matrix. It turns out that the matrix $\Omega_{2 m}(c)$ is not uniquely defined. Indeed, consider the set of matrices

$$
\begin{aligned}
\mathcal{L}:= & \left\{L \in R^{\sigma(n, m) \times \sigma(n, m)}:\right. \\
& \left.L=L^{\prime}, x^{\{m\}^{\prime}} L x^{\{m\}}=0 \forall x \in R^{n}\right\} .
\end{aligned}
$$

Such a set has the following property.

\section{Lemma 2.}

$\mathcal{L}$ is a linear space and its dimension $d_{\mathcal{L}}$ is

$$
d_{\mathcal{L}}=\frac{1}{2} \sigma(n, m)[\sigma(n, m)+1]-\sigma(n, 2 m) .
$$

The above lemma suggests that all the matrices belonging to $\mathcal{L}$ admit a linear parameterization. The proof of Lemma 2 and details on such a parameterization can be found in [7].

\section{Lemma 3.}

Let $\hat{\Omega}_{2 m}(c)$ be any symmetric matrix such that

$$
\hat{w}(x ; c)=x^{\{m\}^{\prime}} \hat{\Omega}_{2 m}(c) x^{\{m\}} .
$$

Let $L(\alpha)$ be a linear parameterization of $\mathcal{L}$ and define

$$
\Omega_{2 m}(c ; \alpha):=\hat{\Omega}_{2 m}(c)+L(\alpha) .
$$

Then,

$\hat{w}(x ; c)=x^{\{m\}^{\prime}} \Omega_{2 m}(c ; \alpha) x^{\{m\}} \quad \forall \alpha \in R^{d_{\mathcal{L}}}, \forall x \in R^{n}$.

Proof. Obvious, since

$$
x^{\{m\}^{\prime}} L(\alpha) x^{\{m\}}=0 \quad \forall \alpha \in R^{d_{\mathcal{L}}}, \forall x \in R^{n} .
$$

Notice that, for a fixed $c$, the symmetric matrix $\Omega_{2 m}(c ; \alpha)$ depends affine linearly on $\alpha$. 
We can now give the main result of this section.

\section{Theorem 1.}

Let

$$
\hat{c}=\sup c
$$

such that

$$
\min _{\alpha} \lambda_{\max }\left[-\Omega_{2 m}(c ; \alpha)\right] \leq 0 .
$$

Then, $\hat{c}$ is a lower bound of $c^{*}$, i.e.,

$$
\hat{c} \leq c^{*}
$$

Proof. From Lemma 1, it is straightforward to verify that problem (5) can be rewritten as

$$
c^{*}=\sup c
$$

such that

$$
\hat{w}(x ; c) \geq 0 .
$$

Now, let $\hat{\alpha}$ be such that

$$
\lambda_{\max }\left[-\Omega_{2 m}(\hat{c} ; \hat{\alpha})\right]=0 .
$$

From Lemma 2 and Lemma 3 it follows that $\hat{w}(x, \hat{c}) \geq 0$ and thus the proof is complete.

The above theorem states that a lower bound for Problem II can be computed via the solution of a one-parameter family of convex optimization problems. Specifically, for a fixed value of the parameter $c$, each problem requires the minimization of the maximum eigenvalue of a symmetric matrix with respect to the vector $\alpha$ (see $(20))$, a problem that can be solved via a standard LMI [6].

To conclude the section, we consider a special class of problems (5) for which only a single LMI problem is needed for computing the lower bound $\hat{c}$. Such a class $\mathcal{C}$ includes polynomials $w(x)$ with two terms, as precisely defined below.

Definition 4. A polynomial $w$ is said to belong to the class $\mathcal{C}$ if

$$
w(x)=v^{(2 l)}(x)+v^{(2 m)}(x)
$$

where $l \in\{0,1\}$.

Such a class has the following property [7].

\section{Lemma 4.}

Let $w \in \mathcal{C}$ be locally positive definite. Then, there exist a positive definite matrix $M_{0}$ and a symmetric matrix $M_{1}$ such that

$$
\Omega_{2 m}(c, \alpha)=c^{l-m} M_{0}+M_{1}+L(\alpha) .
$$

We are now ready to state the next theorem.

\section{Theorem 2.}

Assume that the polynomial $w$ in problem (5) belongs to the class $\mathcal{C}$. Let

$$
\sigma=\min _{\alpha} \lambda_{\max }\left[\left(\hat{M}_{0}^{\prime}\right)^{-1}\left(-M_{1}-L(\alpha)\right)\left(\hat{M}_{0}\right)^{-1}\right]
$$

where $\hat{M}_{0}$ is such that

$$
M_{0}=\hat{M}_{0}^{\prime} \hat{M}_{0}
$$

Then,

$$
\hat{c}=\sigma^{\frac{1}{l-m}}
$$

Proof. It immediately follows from condition (20) and equation (26).

\section{Optimality properties of the LMI-based lower bound}

In the previous section an LMI-based lower bound $\hat{c}$ has been computed. The key step in the development of such a bound is the positivity condition of the matrix $\Omega_{2 m}(c ; \alpha)$. Obviously, such a condition is more stringent than the actual condition of positivity of the corresponding form $\hat{w}(x ; c)$. This fact is responsible for making the lower bound $\hat{c}$ in general suboptimal, i.e., strictly lower than the optimal solution $c^{*}$.

In this section, we give a result for checking if we have indeed $\hat{c}=c^{*}$.

\section{Theorem 3.}

Let $\hat{\alpha}$ be such that

$$
\lambda_{\max }\left[-\Omega_{2 m}(\hat{c} ; \hat{\alpha})\right]=0 .
$$

Then, the following statements are equivalent

$$
\begin{aligned}
& \text { i) } \hat{c}=c^{*} \\
& \text { ii) } \exists \hat{x} \in R^{n}:\left\{\begin{array}{l}
\hat{x}^{\{m\}} \in \operatorname{Ker}\left[\Omega_{2 m}(\hat{c} ; \hat{\alpha})\right] \\
u(\hat{x})=\hat{c}
\end{array}\right. \text {. }
\end{aligned}
$$

Proof. It easily follows from the fact that condition ii) yields the equivalence of positive semidefiniteness of the matrix $\Omega_{2 m}(\hat{c} ; \hat{\alpha})$ and the corresponding form $\hat{w}(x ; \hat{c})=x^{\{m\}^{\prime}} \Omega_{2 m}(\hat{c} ; \hat{\alpha}) x^{\{m\}}$.

\section{Remark 2.}

The theorem above suggests the following simple test for checking optimality of $\hat{c}$. Compute a vector of the null space of $\Omega_{2 m}(\hat{c} ; \hat{\alpha})$ and verify if it is equal to $x^{\{m\}}$ for some $x \in R^{n}$. It can be shown that such a verification amounts to solving a system of $n$ linear equations [7]. 


\section{Application examples}

In this section we discuss two examples concerning the stability margin of control systems [3] and the estimation of the domain of attraction of equilibria [4]. Details on the computational steps (e.g., linear parametrization $L(\alpha)$ ) can be found in [7].

Example 1. Consider the state-space system

$$
\dot{z}(t)=A(x) z(t)
$$

where $z \in R^{n}$ is the state vector and $x=\left(x_{1}, \ldots, x_{n}\right)^{\prime} \in$ $R^{n}$ is the vector of uncertain parameters. Assume that $A(0)$ is a Hurwitz matrix. Then, it is well kwown ([8],[9]) that the $l_{2}$ parametric stability margin is given by

$$
\rho_{2}=\sqrt{\rho^{*}}=\sqrt{\min \left\{\rho_{R}, \rho_{I}\right\}}
$$

where $\rho_{R}$ is the solution of the optimization problem

$$
\begin{gathered}
\rho_{R}=\min _{x \in R^{n}} x_{1}^{2}+x_{2}^{2} \\
\text { subject to } \operatorname{det}[A(x)]=0 .
\end{gathered}
$$

and $\rho_{I}$ is

$$
\rho_{I}=\min _{x \in R^{n}} x_{1}^{2}+x_{2}^{2}
$$

$$
\text { subject to } \quad H_{n-1}[A(x)]=0 \text {. }
$$

If $A(x)$ depends polynomially on $x$, then the problems above are in the form of Problem I, once $W=E_{n}$ and $p(x)=(-1)^{n} \operatorname{det}[A(x)]$ in problem (34) while $p(x)=$ $H_{n-1}[A(x)]$ in problem (35) . To illustrate the computational steps, we consider the following numerical case

$$
A(x)=\left[\begin{array}{lll}
2 x_{1} & 2 x_{1}-4 x_{2}+1 & -x_{1}+5 x_{2} \\
0 & 2 x_{1}+2 x_{2} & 2 x_{1}+1 \\
3 x_{2}-1 & -3 & -3 x_{1}-3
\end{array}\right] .
$$

In this case we have

$$
\begin{aligned}
\operatorname{det}[A(x)]= & -1+2 x_{1}+7 x_{2}-6 x_{1}^{2}+16 x_{1} x_{2}-2 x_{2}^{2} \\
& -12 x_{1}^{3}+6 x_{1}^{2} x_{2}-48 x_{1} x_{2}^{2} \\
H_{2}[A(x)]= & 8-22 x_{1}-2 x_{2}-23 x_{1}^{2}+34 x_{1} x_{2}-45 x_{2}^{2} \\
& -4 x_{1}^{3}+21 x_{1}^{2} x_{2}-35 x_{1} x_{2}^{2} .
\end{aligned}
$$

Figures 2 and 3 show that the optimization problems (34) and (35) have local minima in addition to the global one. Let us consider problem (34). According to Proposition 1 and taking into account the form of $p(x)$, it results

$$
\begin{gathered}
u(x)=x_{1}^{2}+x_{2}^{2} \\
w(x)=\quad 1+8 x_{1}^{2}-60 x_{1} x_{2}-45 x_{2}^{2}+84 x_{1}^{4}-48 x_{1}^{3} x_{2} \\
+388 x_{1}^{2} x_{2}^{2}+728 x_{1} x_{2}^{3}+424 x_{2}^{4}-144 x_{1}^{6} \\
+144 x_{1}^{5} x_{2}-1188 x_{1}^{4} x_{2}^{2}-144 x_{1}^{3} x_{2}^{3} \\
-1944 x_{1}^{2} x_{2}^{4}-2880 x_{1} x_{2}^{5}-900 x_{2}^{6} .
\end{gathered}
$$

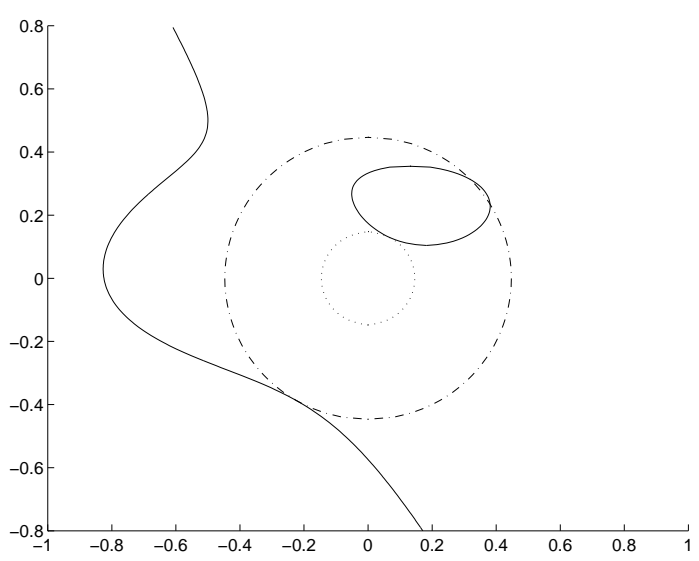

Figure 2: Problem (34): boundary of $p(x)=0$ (solid), global minimum (dotted) and local minimum (dashdot).

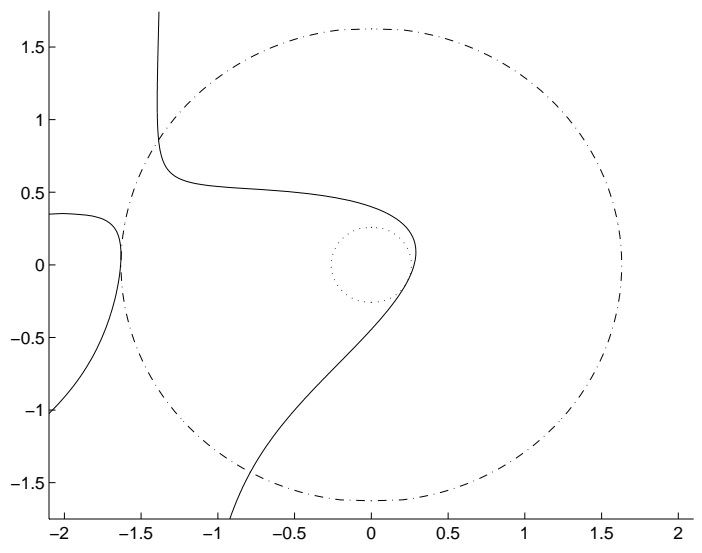

Figure 3: Problem (35): boundary of $p(x)=0$ (solid), global minimum (dotted) and local minimum (dashdot).

Since $n=2$ and $m=3$, Lemma 2 provides $d_{\mathcal{L}}=3$. Using Theorem 1 we obtain

$$
\hat{c}=0.0213 .
$$

This bound is optimal since condition ii) of Theorem 3 holds for

$$
\hat{x}=[0.063,0.131]^{\prime} .
$$

Therefore, we have

$$
\rho_{R}=\hat{c}=0.0213 .
$$

In the same way, we find for problem (35)

$$
\hat{c}=0.0682 \text {. }
$$

The bound $\hat{c}$ is optimal in this case, too. Indeed, we find that condition ii) of Theorem 3 holds for

$$
\hat{x}=[0.234,-0.117]^{\prime} .
$$

Therefore, we have

$$
\rho_{I}=\hat{c}=0.0682
$$


and finally from (32)

$$
\rho_{2}=0.146
$$

Example 2. Consider the state-space system

$$
\dot{x}(t)=f(x(t))
$$

where $x \in R^{n}$ is the state vector and $f$ is a given nonlinear vector field. Assume that the origin in an asymptotically stable equilibrium point. Then, it is well kwown that the largest ellipsoidal estimate of the domain of attraction of the origin, that can be computed via the quadratic Lyapunov function $V(x)=x^{\prime} P x$, is given by $V(x)=\rho^{*}$, where $\rho^{*}$ is the solution of the optimization problem

$$
\begin{aligned}
& \qquad \rho^{*}=\min _{x \in R_{0}^{n}} x_{1}^{2}+x_{2}^{2} \\
& \text { subject to } \quad-x^{\prime} P f(x)-f^{\prime}(x) P x=0 .
\end{aligned}
$$

If $f(x)$ depends polynomially on $x$, then the problem above is in the form of Problem I, once $W=P$ and $p(x)=-x^{\prime} P f(x)-f^{\prime}(x) P x$. To illustrate the computational steps, we consider the following numerical case

$f(x)=\left[\begin{array}{l}-0.5 x_{1}-0.95 x_{1} x_{2}^{2}-0.2 x_{2}^{3} \\ -0.5 x_{2}+2 x_{1}^{3}+0.7 x_{2}^{3}\end{array}\right] ; \quad V(x)=x_{1}^{2}+x_{2}^{2}$.

In this case we have

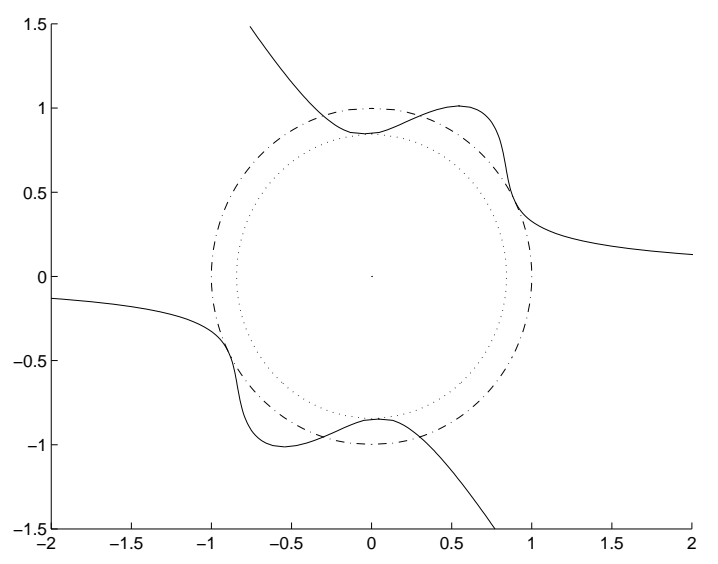

Figure 4: Boundary of $p(x)=0$ (solid), global minimum (dotted) and local minimum (dashdot).

$$
p(x)=x_{1}^{2}+x_{2}^{2}-4 x_{1}^{3} x_{2}+1.9 x_{1}^{2} x_{2}^{2}+0.4 x_{1} x_{2}^{3}-1.4 x_{2}^{4} .
$$

Figure 4 shows that the optimization problem has a local minimum in addition to the global one.

According to Proposition 1, it results

$$
\begin{gathered}
u(x)=x_{1}^{2}+x_{2}^{2} \\
w(x)=x_{1}^{2}+x_{2}^{2}-4 x_{1}^{3} x_{2}+1.9 x_{1}^{2} x_{2}^{2}+0.4 x_{1} x_{2}^{3}-1.4 x_{2}^{4} .
\end{gathered}
$$

It turns out $d_{\mathcal{L}}=1$ and from Theorem 1 we get

$$
\hat{c}=0.710 .
$$

This bound is optimal also in this case. Thus, we have

$$
\rho^{*}=\sqrt{\hat{c}}=0.843 .
$$

\section{Conclusion}

In this paper the problem of determining the minimum euclidean distance of a point from a polynomial surface in $R^{n}$ is considered. As a first result it is shown that a lower bound to the global minimum is achieved via the solution of a one-parameter family of LMIs, once a suitable change of variables is performed. Each LMI problem requires the minimization of the maximum eigenvalue of a symmetric matrix. Successively, a simple test for checking tightness of this lower bound is given.

\section{References}

[1] J.C. Doyle, J.E. Wall, G. Stein, "Performance and robustness analysis for structured uncertainty," Proc. 21st IEEE Conf. on Decision and Control, Orlando, Florida, pp. 629-636, 1982.

[2] J.C. Doyle, " Analysis of feedback systems with structured uncertainties," IEE Proceedings-D Control Theory and Applications, vol. 129, pp. 242-250, 1982.

[3] S.P. Bhattacharyya, H. Chapellat and L. H. Keel, Robust Control: The Parametric Approach, Prentice Hall PTR, NJ, 1995.

[4] R. Genesio, M. Tartaglia, and A. Vicino, "On the estimation of asymptotic stability region. State of the art and new proposals", IEEE Transactions on $\mathrm{Au}$ tomatic Control, vol. 30, pp. 747-755, 1985.

[5] H. D. Chiang and J. S. Thorp, "Stability regions of nonlinear dynamical systems: a constructive methodology", IEEE Transactions on Automatic Control, vol. 34, pp. 1229-1241, 1989.

[6] S. Boyd, L. El Ghaoui, E. Feron and V. Balakrishnan, Linear Matrix Inequalities in System and Control Theory, Siam, Philadelphia, 1994.

[7] G. Chesi, A. Tesi, A. Vicino, R. Genesio, "On convexification of some minimum distance problems," Research Report DSI-19/98, Dipartimento di Sistemi e Informatica, Firenze, 1998.

[8] A. Tesi, A. Vicino, "Robust stability of state space models with structured uncertainties," IEEE Transanctions on Automatic Control, vol. 35, pp. 191-195, 1990.

[9] A.T. Fuller, "Conditions for a Matrix to Have Only Characteristic Roots with Negative Real Parts", Journal of Mathematical Analysis and Applications, 23, pp. 71-98, 1968. 